\title{
Peritoneal tuberculosis presenting as a pelvic mass mimicking ovarian cancer: A case report
}

\author{
DM Casather
}

\section{Introduction}

Abdomino-pelvic tuberculosis is a rare disease involving the peritoneal surfaces, gastrointestinal tract, lymph nodes or the viscera. Because it remains asymptomatic for long periods and there is a lack of reliable investigations to confirm the diagnosis, the exact incidence of abdomino-pelvic tuberculosis is not known. Here, we present a case of pelvic tuberculosis that was referred as an adnexal mass for further evaluation.

\section{Case report:}

A 53-year-old woman complained of abdominal pain and distention for 4 weeks. She had lost 15 $\mathrm{kg}$ during the preceding 3 months. She attained menarche at the age of 12 years and had had regular menstrual periods while in reproductive age. She was a mother of two, both of whom were delivered vaginally. She reached menopause at the age of 52 years. On general examination, she was wasted and on abdominal examination, there was mild abdominal fullness. On abdominal/pelvic ultrasound scan, there was a $50 \mathrm{~mm}$ x $54 \mathrm{~mm}$ sized complex mass in the pelvis. The cancer antigen 125 was $175 \mathrm{IU} / \mathrm{ml}$ (0-35 IU/ ml). After initial evaluation, she underwent diagnostic laparoscopy. Tissue samples were obtained prior to the planned surgical treatment. Subsequent biopsies revealed non-caseating granulomas with no evidence of malignancy. Preliminary acid fast bacilli (AFB) stains were negative. Four weeks later, results from the biopsy culture were positive for Mycobacterium tuberculosis. The patient was subsequently diagnosed with disseminated pelvic tuberculosis and referred for combination therapy.

\section{Conclusion:}

Pelvic tuberculosis should always be considered as a differential diagnosis in a patient with an adnexal mass, ascites or an elevated CA-125.

Keywords: Abdomino-pelvic tuberculosis, Mycobacterium tuberculosis

National Hospital, Kandy, Sri Lanka, 